\title{
Increase in Adverse Health Effects Related to Synthetic Cannabinoid Use
}

\author{
Royal K. Law ${ }^{\star 1}$, Josh Schier ${ }^{1}$, Colleen Martin ${ }^{1}$, Arthur Chang ${ }^{1}$, Amy Wolkin ${ }^{1}$ and Jay \\ Schauben² \\ ${ }^{1}$ Centers for Disease Control and Prevention, Chamblee, GA, USA; ${ }^{2}$ American Association of Poison Control Centers, Alexandria, VA, \\ USA
}

\section{Objective}

The Centers for Disease Control and Prevention analyzed all calls to poison centers about synthetic cannabinoid use from January to May 2015 to identify risk factors and adverse health effects related to this emerging public health threat.

\section{Introduction}

Synthetic cannabinoids include various psychoactive chemicals that are sprayed onto plant material, which is then smoked or ingested to achieve a "high." These products are sold under a variety of names (e.g., synthetic marijuana, spice, K2, black mamba, and crazy clown) and are sold in retail outlets as herbal products and are often labeled not for human consumption. Law enforcement agencies regulate many of these substances; however, manufacturers may frequently change the formulation and mask their intended purpose to avoid detection and regulation.

On April 6, 2015, automated surveillance algorithms via surveillance through the National Poison Data System (NPDS), a web-based surveillance system of all calls to United States (US) poison centers (PCs), identified an increase in calls to PCs related to synthetic cannabinoid use. To identify risk factors and adverse health effects, CDC analyzed all calls to PCs about synthetic cannabinoid use from January to May, 2015.

\section{Methods}

We defined a synthetic cannabinoid call as any human exposure to a PC where use of a synthetic cannabinoid product was known or suggested during January to May 2015. We identified calls using NPDS and compared total and monthly call volumes during the study period to the same months in the previous year. Descriptive statistics for sex, age, most frequent signs and symptoms, medical outcome, and route of exposure were calculated. NPDS medical outcome designations utilized for this study included one of the following: 1) death, 2) major (signs or symptoms that are life-threatening or result in substantial residual disability or disfigurement), 3) moderate (signs and symptoms which are not likely to be life-threatening or result in severe disability), 4) minor (signs or symptoms that are minimally bothersome and generally resolve rapidly with no residual disability or disfigurement), or 5) not followed (the patient likely exhibited only minimal toxicity based on clinical judgment). We identified the most common drugs used when multiple substance use was reported. We compared demographic characteristics of calls reporting more severe medical outcomes (major effect and death) to calls reporting less severe outcomes (moderate effect, minor effect, no effect, not followed).

\section{Results}

During the 2015 study period, PCs reported 3,572 calls related to synthetic cannabinoid use, a $229 \%$ increase from the 1,085 calls during the same January-May period in 2014. The number of monthly calls spiked notably in April (1,501 [42.0\%]) before decreasing nearly to 2014 levels by the end of May. Most calls concerned use among males $(2,882$ [80.7\%]). Among 3,442 (96.4\%) calls where age of the user was recorded, the median age was 26 years (range $=7$ months -72 years). Agitation was the most frequently reported health effect $(1,262$ [35.3\%]), followed by tachycardia $(1,035[29.0 \%])$, drowsiness or lethargy (939 [26.3\%]), vomiting (585 [16.4\%]), and confusion (506, [4.2\%]). Among 2,961 calls for which a medical outcome was reported, the majority had either moderate (1407 [47.5\%]) or minor outcomes (1,095), [37.0\%]). There were $15(0.5 \%)$ reported deaths.

Inhalation by smoking was the most common route of exposure $(2,870[80.3 \%])$, followed by ingestion (698 [19.5\%]). Most reported use was intentional (3,310 [92.7\%]). Among 626 calls reporting use of synthetic cannabinoids in combination with one or more other substances, alcohol was most commonly reported (144 [23.0\%]), followed by plant-derived marijuana (103 [16.5\%]), and benzodiazepines $(69[11.0 \%])$. One of the 15 deaths included reports of multiple substance use.

Males were significantly more likely to have a severe outcome $(88.6 \%)$ than a less severe outcome $(80.1 \%)(p<0.001)$. Age group and severity were significantly associated with each other $(\mathrm{p}<0.001)$; persons aged $30-39$ years and aged $>40$ years were significantly more likely than those aged $10-19$ years to report a severe outcome $(\mathrm{p}=$ 0.001 and $p<0.001$, respectively).

\section{Conclusions}

Increased calls related to synthetic cannabinoid use likely related to availability of new variants suggest that synthetic cannabinoids pose an emerging public health threat. The increase in calls described here suggest a need for greater public health surveillance and awareness and targeted public health messaging.

\section{Keywords}

Synthetic; Marijuana; Cannabinoid; Surveillance; Poison Center

\section{*Royal K. Law}

E-mail: hua1@cdc.gov 